\title{
UNIÓN EUROPEA Y DIPLOMACIA PÚBLICA POST-LISBOA: LOS CASOS DE ARGENTINA, BRASIL Y MÉXICO
}

María Luisa Azpíroz Manero

El Tratado de Lisboa implica una reorganización para dar un mayor impulso a la política y acción exterior de la Unión Europea, incluyendo su diplomacia pública. Este artículo examina la diplomacia pública de la Unión Europea en Argentina, Brasil y México en un contexto post-Lisboa, abordando aspectos como su funcionamiento, fortalezas y debilidades. Para ello se recurre a fuentes académicas, oficiales y, sobre todo, a la información obtenida en entrevistas con personal del Servicio Europeo de Acción Exterior (SEAE), tanto en Bruselas como en las Delegaciones de la Unión Europea en los tres países objeto de estudio. ${ }^{1}$

La diplomacia pública es una actividad diplomática y de comunicación política internacional que busca crear una imagen positiva y/o influencia política, con que contribuya al logro de objetivos de política exterior. Según Joseph Nye, funciona como una herra-

${ }^{1}$ Parte del contenido de la presente investigación, todavía en proceso de preparación, fue presentado en dos congresos internacionales. Véase María Luisa Azpíroz, "European Union Public Diplomacy through the eEAs: The Cases of Argentina, Brazil and Mexico", Global and Regional Powers in a Changing World, FLACsO-ISA Joint International Conference, Buenos Aires, 23-25 de julio de 2014, http://web. isanet.org/Web/Conferences/FLACSO-ISA BuenosAires 2014/Archive/731f6f7ae0d7-4716-910f-ecac99fd15f3.pdf; y, "Diplomacia pública: ¿de la teoría a la práctica?: El caso de la Unión Europea en Brasil”, 1964-2014 autoritarismo, democracia e direitos humanos, IX Encontro da ABCP, Brasilia, 4 a 7 de agosto de 2014, http:// www.encontroabcp2014.cienciapolitica.org.br/resources/anais/14/1403712999_ ARQUIVO_PAPERMARIALUISAAZPIROZ.pdf 
mienta para potenciar el poder blando: un tipo de poder basado en la capacidad de atracción, influencia y persuasión que ocurre de modo indirecto cuando se movilizan recursos como la cultura, los principios, las estrategias de política exterior y las instituciones. ${ }^{2}$ La Unión Europea, precisamente, es una potencia internacional asociada al concepto de poder blando, y su diplomacia pública ha recibido una atención académica creciente en los últimos años. ${ }^{3}$

Resulta importante analizar la diplomacia pública de la Unión Europea como parte de su política y acción exterior en América Latina y, en concreto, en Argentina, Brasil y México. La Unión Europea y América Latina mantienen fuertes lazos históricos, culturales y económicos, así como una Asociación Estratégica Birregional establecida en 1999, cuando se celebra la primera cumbre Unión Europea-América Latina y el Caribe. La Unión Europea es el segundo socio comercial de la región y el más importante inversor extranjero, además de ser, junto con los Estados miembros, un importante donante de ayuda oficial al desarrollo. ${ }^{4}$ En el conjunto de América Latina, Argentina, Brasil y México (los dos último, socios estratégicos de la Unión Europea) destacan por su tamaño, población, peso político y económico.

${ }^{2}$ Cfr. J. Nye, The Future of Power, Nueva York, Public Affairs, 2011; "Public Diplomacy and Soft Power", The Annals of the American Academy of Political and Social Science, 616, 1, 2008, pp. 94-109; y Soft Power: The Means to Success in World Politics, Nueva York, Public Affairs, 2004.

${ }^{3}$ Véase, por ejemplo, M. K. Davis Cross y J. Melissen, European Public Diplomacy: Soft Power at Work, Nueva York, Palgrave Macmillan, 2013; S. Duke, "The European External Action Service and Public Diplomacy”, Discussion Papers in Diplomacy, núm. 127, La Haya, Netherlands Institute of International Relations "Clingendael”, 2013; T. La Porte, "El poder de la Unión Europea en el Gobierno Global: Propuesta para una nueva diplomacia pública”, CPD Perspectives on Public Diplomacy, Los Ángeles, Figueroa Press, 2011.

${ }^{4}$ Cfr. SEAE, "UE-América Latina y el Caribe: una asociación estratégica para el siglo XXI", 2012, http://www.eeas.europa.eu/lac/docs/2012_eu-celac_leaflet_ es.pdf 
1. El ConCepto de diplomacia pública en la Unión Europea: DIVERGENCIAS ENTRE TEORÍA Y PRÁCTICA

Como se menciona al inicio, la diplomacia pública es una actividad diplomática y de comunicación política internacional que busca crear una imagen positiva y/o influencia política, con que contribuya al logro de objetivos de política exterior. Con base en las aportaciones de Javier Noya, ${ }^{5}$ las actividades de diplomacia pública podrían ser clasificadas en cuatro tipos (no excluyentes entre sí) ${ }^{6}$

-Diplomacia mediática. Hace referencia a acciones de información y comunicación estratégica que se desarrollan en el corto plazo. Estas acciones van de las más unilaterales a las más interactivas: discursos, declaraciones, notas de prensa, folletos, artículos publicados en medios institucionales propios o en medios extranjeros, páginas electrónicas, redes sociales, ruedas de prensa, seminarios, eventos mediáticos, apariciones en los medios, concesión de entrevistas. Son acciones desempeñadas principalmente por líderes políticos y sus portavoces, así como por diplomáticos y asesores de prensa de las embajadas.

-Diplomacia cultural. Abarca intercambios y acciones vinculadas con la ciencia, la educación y la cultura (idiomas, literatura, cine, música, arte). Son actividades que se desarrollan principalmente en

${ }^{5}$ Cfr. J. Noya, Diplomacia pública para el siglo XxI: la gestión de la imagen exterior y la opinión pública internacional, Barcelona, Ariel, 2007.

${ }^{6}$ Existen otras clasificaciones compatibles con la de Noya. Nicholas Cull destaca cinco áreas de actividad en la diplomacia pública: escucha (incluyendo el monitoreo de medios y las encuestas de opinión), advocacy (defensa de ideas y políticas), diplomacia cultural, intercambio y emisiones internacionales (medios institucionales). Rhonda Zaharna distingue dos áreas principales de actividad: transferencia de información y construcción de relaciones. Mark Leonard apunta tres dimensiones según el corto, medio y largo plazo: gestión de noticias-advocacy, comunicación estratégica-branding y construcción de relaciones-engagement. Cfr. N. J. Cull, "Public Diplomacy: Taxonomies and Histories", The Annals of the American Academy of Political and Social Science, 616, 2008, pp. 31-54; R. S. Zaharna, "Mapping Out a Spectrum of Public Diplomacy Initiatives: Information and Relational Communication Frameworks", en N. Snow y P. Taylor (eds.), Routledge Handbook of Public Diplomacy, Londres, Routledge, 2002; M. Leonard, Public Diplomacy, Londres, Foreign Policy Centre, 2002. 
el largo plazo. El objetivo es promover el diálogo, el conocimiento y el beneficio mutuo, creando relaciones positivas y duraderas entre ciudadanos de distintos orígenes, así como entre instituciones y ciudadanos extranjeros.

-Diplomacia de nicho. Implica especializarse en áreas funcionales o geográficas. La especialización funcional se realiza seleccionando asuntos o problemas a abordar, y la geográfica incidiendo en áreas geográficas o segmentos específicos de la población. Se realiza a medio y largo plazo. Buenos ejemplos de este tipo de diplomacia pública son la cooperación al desarrollo o la cooperación entre socios.

-Place branding. Consiste en el desarrollo de estrategias de presentación y venta de una imagen atractiva y específica a nivel internacional, ya sea para un país (nation branding), región (region branding) o ciudad (city branding). El objetivo es ayudar a mejorar la economía, los negocios, el comercio exterior y el turismo. Se realiza a medio y largo plazo.

En las escasas ocasiones en que el SEAE menciona el término "diplomacia pública", hace referencia principalmente a acciones de diplomacia mediática, revelando una visión de la actividad más reducida que la visión académica recién expuesta. Por ejemplo, la definición del Manual de Información y Comunicación para las Delegaciones de la Unión Europea en terceros países y organizaciones internacionales afirma que la diplomacia pública incluye elementos como advocacy, ${ }^{7}$ persuasión pública y provisión básica de información con el objetivo final de acentuar la percepción y el conocimiento público de un actor internacional. ${ }^{8}$ De modo similar, en la página electrónica del SEAE, en referencia a la diplomacia pública que forma parte del Instrumento de Cooperación con Países Industrializados (ICI), se afirma que los objetivos son incrementar la visibilidad de la Unión Europea en su totalidad, promover una mejor comprensión de las acciones y posturas de la Unión Euro-

7 "Advocacy" podría traducirse como "defensa de ideas y políticas".

${ }^{8}$ Cfr. División de Comunicación Estratégica del sEaE y Unidad de Comunicación y Transparencia de DEvCO, Information and Communication: Handbook for EU Delegations in Third Countries and to International Organisations, Ref. Ares (2013) 32604, 11/01/2013, p. 3. 
pea y ejercer una influencia positiva en el modo en que la Unión Europea se percibe en países socios. ${ }^{9}$ En el reglamento por el que se establece un Instrumento de Colaboración para la cooperación con terceros países ${ }^{10}$ para el periodo 2014-2020, se menciona el término "diplomacia pública" tres veces, si bien en ningún momento se define qué es. Se considera una actividad a promover para fomentar el conocimiento generalizado y la visibilidad de la Unión, sus valores e intereses. ${ }^{11}$

Así pues, parece que las actividades de diplomacia cultural, diplomacia de nicho y place branding no son concebidas como diplomacia pública por parte de la Unión Europea. En la sede del SEAE afirman que "diplomacia pública" es un término que se comenzó a utilizar hacia 2011, ${ }^{12}$ y confirman que existe cierta indefinición institucional que plantea problemas a la hora de asignar financiación a esta actividad. ${ }^{13}$ En la Delegación de la Unión Europea en Argentina observan que, en términos conceptuales, la Unión Europea asocia la diplomacia pública con actividades de información y comunicación propias de la diplomacia mediática. ${ }^{14}$ No obstante, desde la Delegación de la Unión Europea en México señalan que toda la actividad que realizan y que implica a terceros podría considerarse diplomacia pública. ${ }^{15}$ En efecto, como se verá más adelante, la diplomacia pública de la Unión Europea incluye acciones más allá de la información y la comunicación estratégica.

${ }^{9}$ Cfr. SEAE, "Public Diplomacy", http://eeas.europa.eu/ici/publicdiplomacy/index_en.htm

${ }^{10}$ En inglés, Partnership Instrument for Cooperation with Third Countries (PI).

${ }^{11}$ Cfr. Diario Oficial de la Unión Europea, "Reglamento (UE) No 234/2014 del Parlamento Europeo y del Consejo de 11 de marzo de 2014 por el que se establece un Instrumento de Colaboración para la cooperación con terceros países”, 15 de marzo de 2014, http://eur-lex.europa.eu/legal-content/ES/TXT/PDF/?uri=CE LEX:32014R0234\&from=ES

${ }^{12}$ Entrevista con Ekaterini Makri, Asesora de Prensa, SEAE, ciudad de México, 15 de agosto de 2014.

13 Entrevista con Ignacio Sobrino-Castello, Desk Officer para Brasil, SEAE, Bruselas, 18 de junio de 2014.

${ }^{14}$ Entrevista con Branko Andjic, Asesor de Prensa, SEAe, Buenos Aires, 22 de julio de 2014.

${ }^{15}$ Entrevista citada con Ekaterini Makri. 


\section{Diplomacia PÚBlica POST-Lisboa: REORGANiZACión PARA UNA MAYOR COORDINACIÓN}

El Tratado de Lisboa ${ }^{16}$ establece una personalidad legal única para la Unión Europea ${ }^{17}$ y añade importantes novedades que mejoran la coordinación horizontal (entre instituciones y políticas de la Unión Europea) de su actuación internacional y del desarrollo de su diplomacia pública.

En primer lugar, un presidente fijo y a tiempo completo $^{18}$ asume el rol de la anterior presidencia rotatoria del Consejo Europeo, ${ }^{19}$ que pasa a ser una institución. Eliminando la presidencia rotatoria semestral, que tendía a introducir nuevas prioridades en las relaciones exteriores según los intereses cortoplacistas del Estado miembro a cargo, la Unión Europea crea más continuidad en su representación diplomática, prioridades políticas y mensaje. ${ }^{20}$

${ }^{16}$ Véase Diario Oficial de la Unión Europea, "Tratado por el que se modifican el Tratado de la Unión Europea y el Tratado constitutivo de la Comunidad Europea, firmado en Lisboa el 13 de diciembre de 2007" (2007/C 306/01), http:/ / eur-lex. europa.eu/legal-content/ES/TXT/PDF/?uri=OJ:C:2007:306:FULL\&from=ES

17 De este modo, la Unión Europea refuerza su poder de negociación y se convierte en un actor más eficiente, capaz de expresarse con claridad en la escena internacional. Cfr. EUROPA.EU, "Tratado de Lisboa: llevar a Europa al siglo xxı", http://europa.eu/lisbon_treaty/glance/index_es.htm

${ }^{18}$ El belga Herman Van Rompuy ocupó el puesto (designado por mayoría cualificada del Consejo Europeo para dos años y medio y renovable una vez) desde el 1 de enero de 2010. Su sucesor desde el 1 de diciembre de 2014 es el polaco Donald Tusk.

${ }^{19}$ El Consejo Europeo determina la dirección general y las prioridades de la Unión Europea. No ejerce funciones legislativas. Está compuesto por los Jefes de Estado o de Gobierno de los Estados miembros, junto con su Presidente y el Presidente de la Comisión. El Alto Representante/Vicepresidente participa en su trabajo. No debe confundirse el Consejo Europeo con el Consejo de la Unión Europea, que reúne a los Ministros de los Estados miembros para adoptar legislación y coordinar políticas. A diferencia del Consejo Europeo, el Consejo de la Unión Europea todavía tiene una presidencia rotatoria semestral. C fr. EUROPA. EU, "El Consejo Europeo: una institución official de la UE", http://www.european-council.europa.eu/the-institution?lang=es; "Consejo de la Unión Europea", http://europa.eu/about-eu/institutions-bodies/council-eu/index_es.htm

${ }^{20}$ Cfr. S. B. Rasmussen, "El Servicio Europeo de Acción Exterior: un reto comunicativo para la diplomacia pública de la Unión Europea”, Cuadernos Europeos de Deusto, 44, 2011, pp. 147-166; S. Duke, op. cit. 
En segundo lugar, con objeto de incrementar el peso, coherencia y visibilidad de la acción internacional de la Unión Europea, ${ }^{21}$ se crea el puesto de Alto Representante de la Unión para Asuntos Exteriores y Política de Seguridad/Vicepresidente de la Comisión Europea (AR/VP)..$^{22}$ El puesto multidimensional de AR/vP establece un vínculo entre los dos ejes de la actuación internacional de la Unión Europea: la política exterior y de seguridad común (PESC) y la acción exterior. En el primer caso, participa en el Consejo Europeo (como AR) y en el Consejo de la Unión Europea (como Presidente del Consejo de Asuntos Exteriores). En el segundo caso, es Vicepresidente de la Comisión Europea (con autoridad para coordinar el trabajo de otros comisarios) y responsable de la acción exterior de la Unión Europea. El AR/vp dirige el Servicio Europeo de Acción Exterior (SEAE), servicio diplomático oficial de la Unión Europea desde diciembre de 2010.

El SEAE se compone de la sede principal en Bruselas y de las Delegaciones de la Unión Europea en terceros países y organizaciones internacionales como la oNU. ${ }^{23}$ Es una institución autónoma dentro de la Unión Europea y tiene su propio presupuesto, absorbe las competencias de la extinta Dirección General (DG) de Relaciones Exteriores-DG RELEX y trabaja estrechamente con direcciones generales previamente miembros de la "familia RELEX", ${ }^{24}$ con el Servicio de Instrumentos de Política Exterior (FPI ${ }^{25}$ y con

${ }^{21}$ EUROPA.EU, "Tratado de Lisboa: llevar a Europa al siglo xxı", http:/ / europa.eu/lisbon_treaty/glance/index_es.htm

${ }^{22}$ La británica Catherine Ashton ha ocupado el puesto (mandato de la Comisión por cinco años) desde el 1 de enero de 2010, y el 1 de noviembre de 2014 será sustituida por la italiana Federica Mogherini.

${ }^{23}$ Las Delegaciones de la Unión Europea reemplazan a las Delegaciones de la Comisión Europea, y representan a la Unión Europea en su totalidad.

${ }^{24}$ La "familia RELEX” incluía: DG RELEX (gestionaba y coordinaba a las Delegaciones de la Comisión Europea, a cargo de promover una imagen unificada y los intereses de la Comisión en el extranjero); DG Ampliación; DG Comercio; DG Desarrollo; Oficina de Cooperación EuropeAid (AIDCO) y Oficina Humanitaria de la Comisión Europea (ЕCHO).

${ }^{25}$ El FPI es un nuevo servicio establecido tras el Tratado de Lisboa. Está bajo la autoridad del AR y trabaja en estrecha cooperación con el SEAE (se localizan juntos en la sede de Bruselas) y las Delegaciones de la Unión Europea. Se encarga de im- 
otras direcciones generales que tengan una dimensión de acción externa (como DG Educación y Cultura). Sus empleados provienen de la Comisión (principalmente del Servicio Exterior de la extinta DG RELEX, de DG Desarrollo y Cooperación-DG DEvco ${ }^{26}$ y de DG Comercio), de la Secretaría General del Consejo Europeo y del cuerpo diplomático de los Estados miembros. ${ }^{27}$

El SEAE es la principal institución a cargo de la diplomacia pública de la Unión Europea a nivel comunicativo y de coordinación. La sede del SEAE en Bruselas se encarga del diseño estratégico y de buscar una coordinación horizontal (entre instituciones y políticas de la Unión Europea) y vertical (entre la Unión Europea y los Estados miembros). Las Delegaciones de la Unión Europea se encargan de la diplomacia pública y la coordinación vertical en el terreno. Colaboran en la implementación y promoción de estrategias y acciones de diplomacia pública diseñadas por el SEAE y la Comisión. Pero también disponen de un presupuesto específico para acciones dirigidas a incrementar la visibilidad de la Unión Europea, promover sus valores y políticas. En diplomacia mediática destacan la preparación y promoción de visitas de personalidades de la Unión Europea; la organización de visitas conjuntas de trabajadores de la Delegación y de embajadas de Estados miembros a diferentes estados o provincias; los viajes a Bruselas para periodistas locales y las acciones y eventos dirigidos a multiplicadores de opinión. El $80 \%$ del trabajo de diplomacia pública a nivel comunicativo se hace en las Delegaciones, siendo el asesor de prensa el encargado de coordinar la estrategia general de comunicación, de adaptar los mensajes de la Unión Europea al contexto local y de crear contactos y redes con los medios locales. ${ }^{28}$ En diplomacia cultural destacan actividades como la celebración del

plementar acciones de política exterior en cuatro áreas principales: Política exterior y de seguridad común (PESC), Instrumento en pro de la estabilidad y la paz (ICSP), Misiones de observación electoral (ЕOM) e Instrumento de cooperación con países industrializados (ICI). Cfr. Comisión Europea, "Service for Foreign Policy Instruments (FPI)-About Us", http://ec.europa.eu/dgs/fpi/about/index_en.htm

${ }^{26}$ Desde enero de 2011, DG Desarrollo y AIDco se fusionan en DG DEvCo.

${ }^{27}$ Cfr. S. Duke, op. cit.

${ }^{28}$ Entrevista citada con Ignacio Sobrino-Castello. 
Día de Europa; eventos y programas para universitarios e institutos de secundaria; la organización de concursos de monografías sobre la Unión Europea para estudiantes; la feria Europosgrados; la Semana de Cine Europeo o el Programa de Vistitantes de la Unión Europea. Otras actividades destacadas son la organización de seminarios de cooperación, derechos humanos o medio ambiente. Además, las Delegaciones de la Unión Europea preparan informes políticos, monitorean los medios locales y programas de desarrollo e identifican las audiencias más relevantes con vistas a promover el diálogo, la colaboración y el conocimiento mutuo. ${ }^{29}$

En las Delegaciones de la Unión Europea, los trabajadores entrevistados coinciden en señalar la mejora de la coordinación vertical (entre la Unión Europea y los Estados miembros) que acontece tras el Tratado de Lisboa y la puesta en marcha del SEAE. Una mayor coordinación vertical es importante para dar una imagen de unidad de la Unión Europea, y coadyuva a la eficacia de las acciones y el aprovechamiento de los recursos. Desde la Delegación de la Unión Europea en Argentina confirman que las embajadas de los Estados miembros están más concienciadas de la necesidad de incorporar la dimensión europea y coordinarse con la Unión Europea (aunque unas más que otras). En Argentina, en una época de restricciones financieras, la buena cooperación permite sacar adelante iniciativas como la Semana de Cine Europeo. ${ }^{30}$ En el caso de los socios estratégicos (Brasil y México), las estrategias de diplomacia pública conjunta a nivel local buscan implementar programas comunicativos y culturales en los que participen todas las misiones de la Unión Europea. ${ }^{31}$ Desde la Delegación de la Unión Europea en Brasil destacan que si bien la incorporación de la dimensión europea por parte de los Estados miembros es lenta, se observa una mayor coordinación tras el Tratado de Lisboa. Las reuniones con las misiones de los Estados miembros, que anteriormente se celebraban

${ }^{29}$ Entrevista citada con Branko Andjic; entrevista con Rita Junqueira, Sección Política, Económica y de Comunicación, SEAE, Brasilia, 8 de agosto de 2014; entrevista citada con Ekaterini Makri.

${ }^{30}$ Entrevista con Branko Andjic, Asesor de Prensa, SEAE, Buenos Aires, 22 de julio de 2014.

${ }^{31}$ Entrevista citada con Ignacio Sobrino-Castello. 
en la sede del país que tuviera la presidencia rotatoria de la Unión Europea, ahora se celebran en la Delegación. Además, la red Eunic (Institutos Culturales Nacionales de la Unión Europea) ha tenido un papel importante en la mejora de la coordinación vertical en Brasil, no sólo a nivel de eventos culturales sino también de prensa, ya que en las embajadas los encargados de cultura suelen serlo también de prensa. ${ }^{32}$ En la Delegación de la Unión Europea en México afirman que las estrategias de diplomacia pública conjunta con los Estados miembros comienzan en 2012, siguiendo el ejemplo de Brasil. En un contexto de imagen negativa de la Unión Europea y disminución de recursos debido a la crisis económica, se buscó poner a todos bajo la misma bandera, logrando una mayor incorporación de la dimensión europea y definiendo unos mensajes comunes clave. Se logró coordinar consejos de prensa y grupos de trabajo en diferentes ámbitos (medio ambiente, derechos humanos...), así como incrementar las actividades conjuntas del Jefe de la Delegación con los embajadores de los Estados miembros. ${ }^{33}$ En los tres países, muchas de las actividades de diplomacia cultural son organizadas en conjunto por las Delegaciones y las embajadas de los Estados miembros.

Las Delegaciones de la Unión Europea también destacan la mejora de la comunicación externa e interna que se ha producido tras el Tratado de Lisboa, señalando el importante papel de la División de Comunicación Estratégica y de la herramienta de comunicación interna ÁGora. ${ }^{34}$ En la sede del seAe, la División de Comunicación Estratégica-Servicio de Portavoces ofrece servicios de apoyo al AR/VP y a cargos importantes del SEAE, y elabora contenidos para los medios y el público general. Además, provee a las Delegaciones de la Unión Europea de "flashes de noticias" con "líneas diarias a adoptar" y "puntos defensivos" sobre los asuntos más relevantes, ${ }^{35}$ información de contexto, material de prensa, alertas

${ }^{32}$ Entrevista con Humberto Netto, Asesor de Prensa, SEAE, Brasilia, 8 de agosto de 2014.

${ }^{33}$ Entrevista citada con Ekaterini Makri.

${ }^{34}$ Entrevista citada con Branko Andjic; entrevista citada con Humberto Netto.

${ }^{35}$ Las Delegaciones de la Unión Europea se encargan de distribuir esta información a las embajadas los Estados miembros. 
de eventos, boletines informativos semanales que resumen los principales eventos de la política exterior de la Unión Europea y acceso a las sesiones informativas diarias en Bruselas (por teléfono o streaming). ${ }^{36}$ También crea, en 2011, el Comité de Información de Relaciones Externas (ERIC), que reúne a representantes de las unidades de comunicación de la Comisión para coordinar actividades de información y comunicación entre ellos y con las Delegaciones de la Unión Europea. Y en 2013, junto con DG DEvCo, elabora un manual de información y comunicación para las Delegaciones de la Unión Europea.$^{37}$ ÁGORA es una herramienta informática de uso interno que permite un mejor flujo de información y comunicación entre la sede del SEAE en Bruselas y las Delegaciones de la Unión Europea, así como entre las Delegaciones de la Unión Europea y las embajadas de los Estados miembros (sirve como mecanismo de consulta horizontal entre consejeros comerciales, políticos, asesores de prensa...).

Junto con el SEAE, DG DEvCo juega un papel importante en la diplomacia pública de la Unión Europea: es el principal responsable de la cooperación al desarrollo, uno de los principales activos de la Unión Europea en la escena internacional. La cooperación al desarrollo puede identificarse como diplomacia de nicho, pues implica especializarse en asuntos o problemas a abordar, así como en áreas geográficas y segmentos de población específicos. Además, la cooperación al desarrollo implica una diplomacia pública menos institucional y más social, ya que se suele proceder financiando proyectos diseñados, implementados y comunicados por la sociedad civil (lo que se ha denominado "diplomacia pública por poderes" 38 ). Las Delegaciones de la Unión Europea dan visibilidad a las convocatorias, a la implementación de los proyectos (des-

${ }^{36}$ Cfr. S. Duke, op. cit.

${ }^{37}$ Véase División de Comunicación Estratégica del SEAE y Unidad de Comunicación y Transparencia de DEvCo, Information and Communication: Handbook for EU Delegations in Third Countries and to International Organisations, Ref. Ares (2013) 32604, 11 de enero de 2013.

${ }^{38}$ En inglés, "public diplomacy by proxy". Cfr. S. B. Rasmussen, "The Messages and Practices of the European Union's Public Diplomacy", The Hague Journal of Diplomacy, 5, 2010, pp. 263-287. 
taca la organización de visitas con periodistas a lugares donde se desarrollan proyectos financiados por la Unión Europea) y a sus resultados. Además, implican a la sociedad civil a cargo de los proyectos en el aspecto comunicativo, al que se debe dedicar un porcentaje del presupuesto: el mensaje elaborado debe ser coherente con el de la Unión Europea, informar de la recepción de fondos e incluir los logos de la Unión Europea. Así pues, el esfuerzo por informar y dar visibilidad a la cooperación al desarrollo queda patente en las páginas electrónicas de DG DEvCO, de las Delegaciones de la Unión Europea y de los responsables de cada proyecto.

Desde DG DEvco también consideran que la coordinación vertical (entre la Unión Europea y los Estados miembros) ha mejorado tras el Tratado de Lisboa. A nivel central, la coordinación vertical se busca con reuniones en el Consejo Europeo y en consulta con el Parlamento Europeo. La coordinación es mayor en el terreno, aunque el hecho de que la cooperación al desarrollo de la Unión Europea y los Estados miembros tenga ciclos de programación diferentes complica la implementación de proyectos y la creación de sinergias. ${ }^{39}$ Con objeto de mejorar el impacto y la efectividad de su cooperación al desarrollo, la Unión Europea ha comenzado a diseñar y aplicar ejercicios de programación conjunta en el terreno, liderados por las Delegaciones de la Unión Europea y las embajadas de los Estados miembros, en más de cuarenta países. ${ }^{40}$ Esto está también en línea con la Declaración de París sobre la Eficacia de la Ayuda al Desarrollo (2005) y con el Programa de acción de Accra (2008), que abogan por una efectiva coordinación de los donantes.

En cualquier caso, en el periodo 2014-2020 la cooperación al desarrollo de la Unión Europea experimentará cambios significativos. De acuerdo con la "Agenda para el Cambio", bloques subregionales (como Mercosur) y países que han progresado en el desarrollo

${ }^{39}$ Entrevista con Teresa Barba, Coordinadora de Cooperación para el Desarrollo para Brasil y América Central, Comisión Europea, DG Devco, Bruselas, 27 de mayo de 2014.

${ }^{40}$ Cfr. Comisión Europea, "The EU's Comprehensive Approach to External Conflicts and Crises", Joint Communication to the European Parliament and the Council 11 de diciembre de 2013, p. 11, http:/ /www.eeas.europa.eu/statements/ docs/2013/131211_03_en.pdf 
de sus economías (incluyendo a Argentina, Brasil y México) dejarán de recibir ayuda bilateral de la Unión Europea, aunque continuarán beneficiándose de los programas temáticos y regionales. ${ }^{41}$ En estos países, el Instrumento de Colaboración para la cooperación con terceros países promoverá la dimensión externa de las políticas internas de la Unión Europea y el tratamiento conjunto de desafíos globales. ${ }^{42}$ Además, tanto Brasil como México, que cuentan con sus propias agencias internacionales de cooperación, ${ }^{43}$ tienen especial interés en la cooperación Sur-Sur y en la cooperación triangular. A Brasil le interesa la cooperación con los denominados países Palop (Países Africanos de Língua Oficial Portuguesa) en ámbitos como la agricultura. México se interesa por los países de Centroamérica: cabe señalar el Proyecto Mesoamérica, que promueve el desarrollo integral de los Estados mexicanos del sur y de nueve países mesoamericanos; y el Programa Integrado de Seguridad y Justicia. En estos casos, la Unión Europea puede ejercer un papel fundamental transmitiendo su "know-how" y su conocimiento técnico. ${ }^{44}$

\section{Fortalezas y debilidades de la DiPlomacia pÚblica DE LA UNión EUROPEA}

Los entrevistados en las tres Delegaciones de la Unión Europea coinciden al señalar las principales fortalezas y debilidades de

${ }^{41}$ Cfr. Comisión Europea, "Política de desarrollo de la UE: la Comisión se propone aumentar el impacto de la ayuda concentrándose en menos sectores y en los países más necesitados”, Comunicado de Prensa, Bruselas, 13 de octubre de 2011, http://europa.eu/rapid/press-release_IP-11-1184_es.htm

${ }^{42}$ Cfr. Diario Oficial de la Unión Europea, "Reglamento (UE) No 234/2014 del Parlamento Europeo y del Consejo de 11 de marzo de 2014 por el que se establece un Instrumento de Colaboración para la cooperación con terceros países", 15/03/2014, http://eur-lex.europa.eu/legal-content/ES/TXT/PDF/?uri=CELEX: 32014R0234\&from=ES

43 Véase Agência Brasileira de Coperação (ABC), http://www.abc.gov.br, y Agencia Mexicana de Cooperación Internacional para el Desarrollo (Amexcid) http://amexcid.gob.mx/

${ }^{44}$ Entrevista citada con Teresa Barba. 
la diplomacia pública de la Unión Europea en los países de estudio. El componente cultural es sin duda el mejor activo de la diplomacia pública de la Unión Europea. En la Delegación en Argentina aseguran que, aunque suene trillado, facilita mucho el trabajo insistir en una cultura proveniente de una herencia común; algo que no funcionaría tan bien, por ejemplo, en países como China o Indonesia. Buenos Aires es una ciudad muy cultural, y los interlocutores argentinos conocen y se interesan por la cultura y la educación europea: éstos son, sin duda, los más exitosos recursos de poder blando de la Unión Europea en Argentina. ${ }^{45}$ En Brasil, un país con gran diversidad étnica y cultural, también destacan que el gran fuerte de la Unión Europea es su cultura rica y variada. ${ }^{46}$ Por su parte, en México destacan el papel del Fondo Cultural Mixto México/UE para la colaboración y el diálogo cultural entre México y la Unión Europea. ${ }^{47}$ La colaboración científica y educativa también es señalada como aspecto de gran importancia y éxito de la diplomacia pública de la Unión Europea.

Por el contrario, la escasa visibilidad de la Unión Europea y la falta de recursos (financieros y humanos) se señalan como las mayores dificultades para el desempeño de la diplomacia pública. En la Delegación de la Unión Europea en Argentina anhelan disponer de los recursos con los que cuentan en México, y en México señalan que en Brasil tienen tres veces más recursos que ellos. En Brasil, por su parte, afirman que su limitado presupuesto y recursos suponen uno de sus principales desafíos. Desde la Delegación de la Unión Europea en Argentina mencionan que la transición hacia un nuevo tipo de cooperación bilateral ha llevado a que el personal de cooperación en las Delegaciones se reduzca, lo que lleva a una mayor pérdida de visibilidad de la acción de la Unión Europea en esta área. En el caso de México, se recuerda además que la Unión Europea no puede competir con Estados Unidos ni en influencia

45 Entrevista con Neal Mac Call, Desk Officer para Argentina, SEAE, Bruselas, 18 de junio de 2014; entrevista citada con Branko Andjic.

${ }^{46}$ Entrevista con Humberto Netto, Asesor de Prensa, SEAE, Brasilia, 8 de agosto de 2014.

${ }^{47}$ Entrevista citada con Ekaterini Makri. 
ni en importancia. ${ }^{48}$ Frente a estas dificultades, hay también bastante coincidencia respecto a la necesidad de racionalizar recursos y dirigir las actividades de diplomacia pública a targets muy específicos, a grupos de interés con los que se busca articular diálogo y conocimiento mutuo. Ese target está compuesto por creadores y multiplicadores de opinión actuales y futuros: se encuentran en los medios, el sector político, económico y cultural, la vida pública, las generaciones jóvenes y los representantes de la sociedad civil.

En Argentina apuntan también la dificultad que se presenta a la hora de explicar la identidad institucional de la Unión Europea y cómo funciona, dada su complejidad. Además, las personalidades políticas de los Estados miembros siguen teniendo, en general, más visibilidad y alcance que las europeas. ${ }^{49}$ Por último, desde México se recuerda la dificultad de medir cambios en la opinión pública a largo plazo, lo que supone una debilidad de la diplomacia pública en términos generales. ${ }^{50}$

\section{REACCiONES A LOS MENSAJES ClAVE SEGÚN EL CONTEXTO LOCAL}

Los mensajes de la diplomacia pública de la Unión Europea tratan de comunicar y exportar sus propios valores, principios y políticas, eligiendo las cuestiones en que se centran según el contexto del lugar al que se dirigen. Además de la mencionada cultura común, las entrevistas en el SEAE destacan otras cinco cuestiones clave promovidas por el mensaje de la diplomacia pública de la Unión Europea: el libre comercio, la integración regional, la cohesión social, el medio ambiente y los derechos humanos.

La eliminación de barreras al comercio internacional es uno de los objetivos fundamentales que guían la actuación internacional de la Unión Europea . ${ }^{51}$ La Unión Europea defiende el libre comercio como clave para generar riqueza y desarrollo, lo que implica la

\footnotetext{
${ }^{48}$ Loc. cit.

${ }^{49}$ Entrevista citada con Branko Andjic.

${ }^{50}$ Entrevista citada con Ekaterini Makri.

${ }^{51}$ Véase el artículo 10A del Tratado de Lisboa.
} 
progresiva abolición de restricciones al comercio internacional y a la inversión directa extranjera, así como la reducción de barreras aduaneras y de otro tipo. La Unión Europea también promueve la integración regional y las relaciones interregionales en el resto del mundo. Se presenta a sí misma como modelo de integración regional, ya que su propia experiencia demuestra la contribución de su modelo regional a la paz, la estabilidad política, la prosperidad económica y el bienestar social. El pluralismo y las contradicciones que surgen de la diversidad se pueden ver como un inconveniente, pero también como una riqueza: el modo en que la Unión Europea logra gestionarlos y avanzar hacia una mayor integración constituye una práctica a exportar a otros grupos regionales. ${ }^{52}$ La integración regional se presenta también como positiva para crear sinergias y tener una voz más fuerte en el gobierno global.

La promoción del libre comercio y la integración regional se encuentra con dificultades en Argentina y Brasil, tanto en el seno de Mercosur como a nivel bilateral. Las negociaciones para un Acuerdo de Asociación Interregional Unión Europea-Mercosur, ${ }^{53}$ que implicaría el establecimiento de un área de libre comercio, se ven dificultadas por diferencias persistentes en el capítulo comercial: desde Mercosur se critican los subsidios de la Unión Europea a la producción y exportación de productos agrícolas, y desde la

${ }^{52}$ Cfr. Agora Forum, "The European Union External Action Service in Times of Crisis and Change: Public Diplomacy and Discourse", European Policy Brief, 2013; T. La Porte, "El poder de la Unión Europea en el Gobierno Global: Propuesta para una nueva diplomacia pública", CPD Perspectives on Public Diplomacy, Los Ángeles, Figueroa Press, 2011.

53 Actualmente, las relaciones Unión Europea-Mercosur se guían por el Acuerdo Marco Interregional de Cooperación de 1995 (en vigor desde 1999). Es un acuerdo que se basa en tres pilares: diálogo político, cooperación y liberalización comercial mutua. Incluye una "cláusula democrática" (concerniente al respeto a valores comunes) y una "cláusula evolutiva" (sobre la posibilidad de completar e incrementar el nivel de cooperación). El objetivo es contribuir a la integración regional y, en una segunda fase, negociar un Acuerdo de Asociación Interregional que implique también el establecimiento de un área de libre comercio. Cfr. S. Santander, "La légitimation de l'Union européenne par l'exportation de son modèle d'intégration et de gouvernance régionale: Le cas du marché commun du sud", Etudes Internationales, 32, 1 de marzo de 2001, pp. 51-76. 
Unión Europea se pide una mayor liberalización de inversiones, servicios y mercados públicos. ${ }^{54} \mathrm{~A}$ nivel bilateral, Argentina ha aumentado sus medidas proteccionistas en los últimos años, complicando las relaciones económicas y comerciales con la Unión Europea: basta recordar la crisis de Repsol-YPF de 2012, que "congeló" las relaciones bilaterales durante un año. ${ }^{55}$ Brasil, por su parte, protege su modelo económico desarrollista, que busca reducir la vulnerabilidad nacional y mantener la autonomía en la formulación de políticas industriales y financieras. ${ }^{56}$ Esta estrategia económica ha funcionado en Brasil gracias a su tamaño continental y a su enorme mercado doméstico. ${ }^{57}$

La integración regional en Mercosur no ha llegado al nivel que desearía la Unión Europea. Tras dos décadas de colaboración, Mercosur no ha evolucionado lo suficiente en la consolidación de su integración regional: no ha finalizado el área de libre comercio y la unión aduanera, no ha creado un cuerpo de negociación colectiva y no ha adoptado legislación común en áreas sujetas a negociaciones (servicios, inversiones, compras gubernamentales). ${ }^{58}$ Como en el resto de América Latina, el regionalismo de Mercosur es más intergubernamental que supranacional, lo que complica la existencia de instituciones comunes fuertes, de una identidad común y de un apoyo social suficiente, dejando el regionalismo a merced de los Estados miembros. ${ }^{59} \mathrm{El}$ apoyo a la integración regional por parte

${ }^{54}$ Cfr. S. Santander, "Les enjeux du sommet de Madrid", Revue Nouvelle, 4, 2010, pp. 11-14.

55 Entrevista citada Neal Mac Call.

${ }^{56}$ Cfr. S. F. Turcotte, "Le Brésil de Lula et l'Amérique du Sud: L'impossible construction d'un status de puissance régionale", en S. Santander (ed.), Le partenariat stratégique Union Européenne-Amérique Latine dans un monde en mutation: quelles évolutions et perspectives?, Revue $\mathrm{n}^{\circ} 2 / 2007$, Bruselas: Centre d'Étude des Relations entre l'Union Européenne et l'Amérique Latine, 2007.

${ }^{57}$ Entrevista con Erik Von Pistohlkors, Sección Política, Económica y de Comunicación, SEAE, Brasilia, 8 de agosto de 2014.

${ }^{58}$ Cfr. S. Santander, "L'Atlantique Sud dans l'agenda extérieur de l'UE. Le cas des relations avec le Brésil”, en D. Brunelle, Communautés Atlantiques: asymétries et convergences, Montréal, Éditions IEIM, 2012.

${ }^{59}$ Cfr. J. A. Sanahuja, "L'UE et l'intégration régionale en Amérique Latine. La nécessité d'adopter une nouvelle stratégie", en S. Santander (ed.), Le partenariat 
de Argentina ha ido variando según la presidencia de turno. Néstor Kirchner dio relevancia a la profundización de Mercosur y a sus relaciones con Sudamérica y el mundo, ${ }^{60}$ pero la integración regional de Mercosur no ha formado parte de las prioridades de Cristina Fernández de Kirchner. Con vistas a reforzar su papel de potencia regional sudamericana, Brasil defiende la integración regional en Mercosur, pero también protege su modelo económico desarrollista. Es por ello que se resiste a una mayor supranacionalidad en la medida en que ésta pueda limitar su capacidad de reacción frente a choques económicos externos. La puesta en marcha de la Comunidad Sudamericana de Naciones en 2004, que pasa a ser la Unión de Naciones Sudamericanas (Unasur) en 2008, se ha entendido como una apuesta brasileña por "agrandar" en lugar de "profundizar" Mercosur, con un proyecto poco institucionalizado y centrado en favorecer la integración física sudamericana, enmarcando la liberalización de los intercambios comerciales y las inversiones intrarregionales. ${ }^{61}$ Más allá de las dificultades que encuentra en su promoción del libre comercio y la integración regional, la Unión Europea es consciente de la evolución progresiva de las relaciones internacionales hacia un mundo multipolar e interdependiente donde ganan peso las potencias emergentes. Por eso, desde 2003 comienza a establecer asociaciones estratégicas con países considerados potencias políticas y económicas a nivel global: entre dichos países se encuentran Brasil (2007) y México (2008). La Asociación Estratégica con Brasil no implica que la Unión Europea renuncie a las negociaciones para un Acuerdo de Asociación Interregional con Mercosur, sino que actúa de modo pragmático y busca impulsar las relaciones bilaterales con un país de peso a nivel global. ${ }^{62}$ Brasil

stratégique Union Européenne-Amérique Latine dans un monde en mutation: quelles évolutions et perspectives?, Revue $\mathrm{n}^{\circ} 2 / 2007$, Bruselas, Centre d'Étude des Relations entre l’Union Européenne et l'Amérique Latine, 2007.

${ }^{60}$ Cfr. S. F. Turcotte, "Le Brésil de Lula et l'Amérique du Sud".

${ }^{61}$ Loc. cit.

${ }^{62}$ Entrevista con Adrianus Koetsenruijter, Encargado de la División de países del Mercosur, sEAE, Bruselas, 27 de mayo de 2014. 
es, además, el miembro de los BRICs que más coopera con la Unión Europea en los foros multilaterales. ${ }^{63}$

A diferencia de Argentina y Brasil, la promoción del libre comercio no encuentra resistencia en las relaciones de la Unión Europea con México. De hecho, entre los pilares del Acuerdo Global Unión Europea-México (en vigor desde 2000) está una asociación económica que incluye la creación de un área de libre comercio. La integración regional no ha sido objeto de la diplomacia pública de la Unión Europea en México, ya que hasta recientemente ${ }^{64}$ este país no ha pertenecido a ningún bloque regional latinoamericano, sino al bloque económico creado en 1994 con el Tratado de Libre Comercio de América del Norte (TLCAN). ${ }^{65}$

La Unión Europea apuesta por el impulso de políticas públicas para la cohesión social en América Latina, como vía para reducir las desigualdades, lograr un mayor desarrollo económico, bienestar social y seguridad. El medio ambiente y el cambio climático son cuestiones prioritarias en la relación de la Unión Europea con terceros países y en su concepto de desarrollo sostenible. También son aspectos clave en la Asociación Estratégica de la Unión Europea con Brasil y México. Por último, la universabilidad e indivisibilidad de los derechos humanos y las libertades fundamentales se mencionan en el artículo 10A del Tratado de Lisboa como principios que la Unión Europea pretende fomentar y consolidar en el resto del mundo.

Aunque Argentina no alcanza el nivel de Brasil o México en términos macroeconómicos o de influencia en los foros globales,

${ }^{63}$ Entrevista citada con Erik Von Pistohlkors.

${ }^{64}$ Junto con Chile, Colombia y Perú, México forma parte del bloque comercial latinoamericano Alianza del Pacífico, creado en 2011. Aunque no sea un bloque de integración regional, existe una agenda política, pues la componen países favorables al libre mercado. Entrevista con Philipp Oliver Gross, Responsable de Relaciones Internacionales para México, SEAE, Bruselas, 18 de junio de 2014.

${ }^{65}$ Pese a su localización geográfica y pertenencia al TLCAN, la web del SEAE excluye a México del apartado "Relaciones de la UE con América del Norte", donde figuran sólo Canadá y Estados Unidos de América. Así pues, la Unión Europea parece identificar a México más como país latinoamericano que norteamericano. Véase SEAE, "Relaciones de la UE con América del Norte", http:/ / eeas.europa.eu/ na/index_es.htm 
presenta un mayor índice de desarrollo humano, ${ }^{66}$ menos problemas de seguridad y de pobreza extrema y estructural. Además, en Argentina hay un mayor progresismo en cuestiones sociales y la sociedad civil se muestra activa y desarrollada. Así pues, la cohesión social no es un asunto de máxima relevancia, pero sí se incide en la defensa de los derechos humanos, que tiene mucha importancia por lo que concierne a la impunidad de los crímenes de la dictadura militar. ${ }^{67}$ Las cuestiones clave para el mensaje de la Unión Europea en Brasil son los derechos humanos, el medioambiente y la deforestación. ${ }^{68}$ La asociación estratégica garantiza un diálogo y colaboración permanente a este respecto, si bien el grado de implicación de Brasil varía: en el caso de los derechos humanos en las prisiones, por ejemplo, su colaboración es menor. ${ }^{69}$ Las cuestiones destacadas por la Unión Europea en México son las referidas al medio ambiente-cambio climático (donde existe un gran acuerdo a nivel político), a los derechos humanos (la impunidad, la violencia en general y contra defensores de derechos humanos y periodistas son especialmente preocupantes $^{70}$ ) y a la cohesión social (el Laboratorio de Cohesión Social Unión Europea-México trata de impulsar políticas públicas en desarrollo social). Si bien los derechos humanos y la cohesión social se promueven a nivel de diálogo político y cooperación, resolverlos depende fundamentalmente de la voluntad política mexicana.

${ }^{66}$ En 2013, Argentina está en el puesto 49 del Índice de Desarrollo Humano de Naciones Unidas, formando parte (aunque en último lugar) del grupo de países con "desarrollo humano muy alto". México (en el puesto 71) y Brasil (en el puesto 79) están en el grupo de países con "desarrollo humano alto". Cfr. Naciones Unidas. Programa de las Naciones Unidas para el Desarrollo, "Sostener el progreso humano: reducir vulnerabilidades y construir resiliencia”, Informe sobre desarrollo humano 2014, pp. 175-179, http://hdr.undp.org/sites/default/files/ reports/14/hdr2013_en_complete.pdf

${ }^{67}$ Entrevista citada con Neal Mac Call.

${ }^{68}$ Entrevista citada con Erik Von Pistohlkors.

${ }^{69}$ Loc. cit.

${ }^{70}$ Entrevista citada con Philipp Oliver Gross. 


\section{DiploMacia MEdiÁtica: COMUNICACIÓN Y RELACIÓN CON LOS MEDIOS LOCALES}

Además de adaptar el mensaje de la Unión Europea al contexto local, las Delegaciones de la Unión Europea deben conocer bien cómo funcionan los medios locales y tener una red fiable de contactos y periodistas.

En Argentina, pese a algunos intentos gubernamentales por controlar ciertos grupos mediáticos, hay bastante libertad de expresión. ${ }^{71}$ La prensa es además muy combativa y sesgada en pro o en contra del gobierno. La Delegación de la Unión Europea convoca a periodistas a reuniones informales para explicarles las distintas políticas europeas y crear redes de confianza con vistas a la futura cobertura de eventos y proyectos. También se han comenzado a hacer visitas institucionales a medios de comunicación, junto con representantes de los Estados miembros. ${ }^{72}$

En Brasil el interés por los temas internacionales es bajo, por lo que la cobertura que logre el mensaje de la Unión Europea dependerá en gran medida de su conexión con cuestiones que en Brasil consideran relevantes para el país (como las relaciones comerciales y con Mercosur). El trabajo del asesor de prensa, su conocimiento de la cultura brasileña y su red de contactos son fundamentales. No obstante, ha de tenerse en cuenta que, dado su tamaño continental, los periódicos son más importantes a nivel regional que nacional. Además, la capacidad de acción y alcance de la Delegación se ve limitada por su localización en Brasilia, ya que muchos de los contactos importantes se encuentran en São Paulo. ${ }^{73}$ Desde la Delegación también subrayan la importancia de una buena capacitación para comunicar eficientemente y tratar con los medios brasileños: de hecho, en noviembre de 2013 se impartió un taller sobre este tema a los embajadores de Estados miembros en Brasil.

${ }^{71}$ Entrevista citada con Neal Mac Call.

${ }^{72}$ Entrevista citada con Branko Andjic.

${ }^{73}$ Entrevista con Humberto Netto, Asesor de Prensa, SEAE, Brasilia, 08/08/2014. 
En México la libertad de expresión se ve limitada por el problema de la violencia contra los periodistas. La prensa local se centra sobre todo en los problemas internos de México, como la corrupción o el narcotráfico, pero les interesa la postura de la Unión Europea al respecto. La Delegación de la Unión Europea destaca la coordinación y la buena estrategia de comunicación conjunta que existe con los Estados miembros, a fin de explicar a los medios cuestiones y políticas de la Unión Europea y dar a conocer la relación Unión Europea-México. Por ejemplo, el Jefe de la Delegación y los embajadores de los Estados miembros han firmado artículos conjuntos para la prensa mexicana con motivo del Día de Europa (9 de mayo), respecto a la crisis económica y a la crisis de Ucrania. También han acudido juntos a programas de emisoras de radio nacionales y universitarias. ${ }^{74}$

\section{LA COOPERACIÓN AL DESARROLLO COMO HERRAMIENTA DEL PODER BLANDO DE LA UNIÓN EUROPEA}

La cooperación al desarrollo es un elemento importante en la diplomacia pública de la Unión Europea, pues le aporta su componente más social y promociona su mensaje de un modo tangible, mediante proyectos y actividades concretas. Por ejemplo, mediante actividades de diplomacia cultural (como el programa de intercambio educativo Erasmus Mundus), proyectos de ayuda a las pymes (como el Programa de Competitividad e Innovación-PRocei Unión Europea-México), a la integración regional (como el proyecto Biotech para el desarrollo de una plataforma regional de investigación y desarrollo de biotecnologías en Mercosur), actividades de concienciación sobre los derechos humanos (como la ayuda al Máster Internacional en Derechos Humanos y Democratización en Argentina), de cuidado del medio ambiente (como el proyecto para la reducción de la deforestación en São Felix do Xingu, en Brasil), o para impulsar políticas

${ }^{74}$ Entrevista citada con Ekaterini Makri. 
sociales (como el Laboratorio de Cohesión Social Unión Europea-México).

No obstante, la repercusión de la cooperación al desarrollo de la Unión Europea en términos de poder blando no está tan clara. Desde la Delegación de la Unión Europea en Argentina lamentan que, pese a la alta participación de este país en los programas científicos y de intercambio educativo, la cooperación al desarrollo de la Unión Europea no sea apenas conocida a nivel local: se trata de un tema poco atractivo para los medios, siempre más interesados en cuestiones políticas y económicas. ${ }^{75}$ Un mayor presupuesto para visibilidad podría mejorar esta situación, ya que la sociedad civil argentina es activa y está bastante desarrollada. ${ }^{76}$ En la Delegación de la Unión Europea en México afirman que la cooperación al desarrollo sí interesa a los medios, y que el problema está más en las dificultades que tiene la Delegación para seguir y dar visibilidad a proyectos que se desarrollan a largo plazo. ${ }^{77}$ Desde la Delegación de Brasil consideran que en este país la cooperación al desarrollo no tiene el efecto de influencia política que sí puede tener en países menos desarrollados, y apuntan a la cooperación entre iguales como fuente de poder blando para la Unión Europea en Brasil. La experiencia de la Unión Europea, con sus aciertos y errores, puede aportar mucho a Brasil en cuestiones como la economía verde, el envejecimiento de la población, la sanidad o la educación. También de cara a la incipiente cooperación triangular en los países PALOp. ${ }^{78} \mathrm{El}$ "Instrumento de Colaboración para la cooperación con terceros países" que se va a aplicar en la cooperación de la Unión Europea con Argentina, Brasil y México va más en esta línea de tratamiento conjunto de desafíos globales, intercambio de "know-how" y buenas prácticas que en la línea de la tradicional cooperación al desarrollo.

${ }^{75}$ Entrevista citada con Branko Andjic.

${ }^{76}$ Loc. cit.

${ }^{77}$ Entrevista citada con Ekaterini Makri.

${ }^{78}$ Entrevista citada con Erik Von Pistohlkors. 


\section{Conclusiones}

La investigación realizada permite arrojar algunas conclusiones respecto a la diplomacia pública de la Unión Europea en Argentina, Brasil y México, en un contexto post-Lisboa.

El concepto oficial de diplomacia pública del SEAE apunta únicamente a actividades de diplomacia mediática, pero en la práctica la Unión Europea desarrolla también, de modo notable, otros tipos de diplomacia pública (cultural, de nicho).

El Tratado de Lisboa aporta novedades que mejoran y dan un mayor impulso a la diplomacia pública de la Unión Europea en los países de estudio. Las novedades institucionales introducidas para mejorar la coordinación horizontal de la actuación internacional de la Unión Europea (Presidente del Consejo Europeo, AR/VP, SEAE) mejoran también la coordinación horizontal de su diplomacia pública. La coordinación vertical de la diplomacia pública también es mayor: la dimensión europea está más presente, y la Unión Europea realiza más actividades conjuntas con los Estados miembros, logrando más impacto y ahorrando costes. En el aspecto comunicativo, se destaca la relevancia de la División de Comunicación Estratégica y la herramienta de comunicación interna ÁGORA.

La gran fortaleza de la diplomacia pública de la Unión Europea es el atractivo de su componente cultural, educativo y científico. Su gran debilidad, la falta de visibilidad, presupuesto y personal. Es por ello que las actividades se centran en targets muy específicos, compuestos por creadores y multiplicadores de opinión actuales y futuros.

Los mensajes más destacados por la diplomacia pública de la Unión Europea hacen referencia a la herencia cultural común, el comercio libre, la integración regional, la cohesión social, el medio ambiente y los derechos humanos. La relevancia que se otorga a estos mensajes, su materialización en proyectos y actividades concretas, y la aceptación y colaboración que logren dependen del contexto local.

Las Delegaciones de la Unión Europea son las principales encargadas de las tareas de comunicación. Se coordinan con la sede central del SEAE y con los Estados miembros, conocen cómo 
funcionan los medios locales y crean redes fiables de contactos y periodistas.

La cooperación al desarrollo es un activo importante de la diplomacia pública de la Unión Europea, pues le proporciona su dimensión más social y tangible. No obstante, su repercusión en términos de poder blando se ve limitada por la falta de interés de los medios y por los recursos limitados para visibilidad y seguimiento de las Delegaciones. Además, la cooperación al desarrollo se va a sustituir por una cooperación entre socios, en la que la ayuda bilateral se sustituye por la cooperación triangular, el tratamiento conjunto de desafíos globales, el intercambio de "know-how" y de buenas prácticas. Queda por ver si este cambio de cooperación conllevará mayor visibilidad pública y repercusión en términos de poder blando.

\section{Bibliografía}

Agora Forum, "The European Union External Action Service in Times of Crisis and Change: Public Diplomacy and Discourse", European Policy Brief, 2013.

Azpíroz, María Luisa, "European Union Public Diplomacy through the EEAS: the cases of Argentina, Brazil and Mexico", Global and Regional Powers in a Changing World, FLACSO-ISA Joint International Conference, Buenos Aires, 23-25 de julio de 2014, http://web.isanet.org/ Web/Conferences/FLACSO-ISA BuenosAires 2014/Archive/731f6 f7a-e0d7-4716-910f-ecac99fd15f3.pdf

— - "Diplomacia pública: ¿de la teoría a la práctica?: El caso de la Unión Europea en Brasil”, 1964-2014 autoritarismo, democracia e direitos humanos, IX Encontro da ABCP, Brasilia, 4 a 7 de agosto de 2014, http: //www.encontroabcp2014.cienciapolitica.org.br/resources/ana is/14/1403712999_ARQUIVO_PAPERMARIALUISAAZPIROZ.pdf

Cull, N. J., "Public Diplomacy: Taxonomies and Histories", The Annals of the American Academy of Political and Social Science, 616, 2008, pp. 31-54.

Davis Cross, M. K. y J. Melissen, European Public Diplomacy: Soft Power at Work, Nueva York, Palgrave Macmillan, 2013.

Duke, S., "The European External Action Service and Public Diplomacy", 
Discussion Papers in Diplomacy, núm. 127, La Haya, Netherlands Institute of International Relations “Clingendael”, 2013.

La Porte, T., "El poder de la Unión Europea en el Gobierno Global: Propuesta para una nueva diplomacia pública”, CPD Perspectives on Public Diplomacy, Los Ángeles, Figueroa Press, 2011.

Leonard, M., Public Diplomacy, Londres, Foreign Policy Centre, 2002.

Noya, J., Diplomacia pública para el siglo XxI: la gestión de la imagen exterior y la opinión pública internacional, Barcelona, Ariel, 2007.

Nye, J., The Future of Power, Nueva York, Public Affairs, 2011.

-, "Public Diplomacy and Soft Power", The Annals of the American Academy of Political and Social Science, 616, 1, 2008, pp. 94-109.

, Soft Power: The Means to Success in World Politics, Nueva York, Public Affairs, 2004.

Rasmussen, S. B., "El Servicio Europeo de Acción Exterior: un reto comunicativo para la diplomacia pública de la Unión Europea”, Cuadernos Europeos de Deusto, 44, 2011, pp. 147-166.

, "The Messages and Practices of the European Union's Public Diplomacy", The Hague Journal of Diplomacy, 5, 2010, pp. 263-287.

Sanahuja, J. A., "L’UE et l'intégration régionale en Amérique Latine. La nécessité d'adopter une nouvelle stratégie”, en S. Santander (ed.), Le partenariat stratégique Union Européenne-Amérique Latine dans un monde en mutation: quelles évolutions et perspectives?, Revue $\mathrm{n}^{\circ} 2 / 2007$, Bruselas, Centre d'Étude des Relations entre l'Union Européenne et l'Amérique Latine, 2007.

Santander, S., "L’Atlantique Sud dans l'agenda extérieur de l'UE. Le cas des relations avec le Brésil”, en D. Brunelle, Communautés Atlantiques: asymétries et convergences, Montréal, Éditions IEIM, 2012.

—. "Les enjeux du sommet de Madrid", Revue Nouvelle, 4, 2010, pp. 11-14.

-, "La légitimation de l'Union européenne par l'exportation de son modèle d'intégration et de gouvernance régionale: Le cas du marché commun du sud", Études Internationales, 32, 1 de marzo de 2001, pp. 51-76.

Turcotte, S. F., "Le Brésil de Lula et l'Amérique du Sud: L’impossible construction d'un status de puissance régionale”, en S. Santander (ed.), Le partenariat stratégique Union Européenne-Amérique Latine dans un monde en mutation: quelles évolutions et perspectives?, Revue $\mathrm{n}^{\circ} 2 / 2007$, 
Bruselas, Centre d'Étude des Relations entre l'Union Européenne et l'Amérique Latine, 2007.

Zaharna, R. S., "Mapping Out a Spectrum of Public Diplomacy Initiatives: Information and Relational Communication Frameworks", en N. Snow y P. Taylor (eds.), Routledge Handbook of Public Diplomacy, Londres, Routledge, 2002.

\section{Fuentes oficiales}

Comisión Europea, "Service for Foreign Policy Instruments (FPI)-About Us", http://ec.europa.eu/dgs/fpi/about/index_en.htm , "The EU's Comprehensive Approach to External Conflicts and Crises", Joint Communication to the European Parliament and the Council 11/12/2013, en http://www.eeas.europa.eu/statements/ docs/2013/131211_03_en.pdf

"Política de desarrollo de la UE: la Comisión se propone aumentar el impacto de la ayuda concentrándose en menos sectores y en los países más necesitados", Comunicado de Prensa, Bruselas, 13/10/2011, http://europa.eu/rapid/press-release_IP-11-1184_es.htm

Diario Oficial de la Unión Europea, "Reglamento (UE) No 234/2014 del Parlamento Europeo y del Consejo de 11 de marzo de 2014 por el que se establece un Instrumento de Colaboración para la cooperación con terceros países", 15/03/2014, http://eur-lex.europa.eu/legal-content/ES/TXT/PDF/?uri=CELEX:32014R0234\&from=ES

-, "Tratado por el que se modifican el Tratado de la Unión Europea y el Tratado constitutivo de la Comunidad Europea, firmado en Lisboa el 13 de diciembre de 2007" (2007/C 306/01), http://eurlex.europa.eu/legal-content/ES/TXT/PDF/?uri=OJ:C:2007:306:FU LL\&from $=\mathrm{ES}$

División de Comunicación Estratégica del SEAE y Unidad de Comunicación y Transparencia de DEvCO, Information and Communication: Handbook forEUDelegations in Third Countries and to International Organisations, Ref. Ares (2013) 32604, 11/01/2013.

EUROPA.EU, "Tratado de Lisboa: llevar a Europa al siglo xxı", http:// europa.eu/lisbon_treaty/glance/index_es.htm 
, "El Consejo Europeo: una institución oficial de la UE", http:// www.european-council.europa.eu/the-institution?lang=es

_ _ "Consejo de la Unión Europea", http://europa.eu/about-eu/ institutions-bodies/council-eu/index_es.htm

Naciones Unidas. Programa de las Naciones Unidas para el Desarrollo, "Sostener el progreso humano: reducir vulnerabilidades y construir resiliencia", Informe sobre desarrollo humano 2014, pp. 175-179, http://hdr.undp.org/sites/default/files/reports/14/hdr2013_en_ complete.pdf

SEAE, "UE-América Latina y el Caribe: una asociación estratégica para el siglo xxı", 2012, http://www.eeas.europa.eu/lac/docs/2012_eu-celac_leaflet_es.pdf

—_, "Public Diplomacy", http://eeas.europa.eu/ici/publicdiplomacy/index_en.htm

__, "Relaciones de la UE con América del Norte", http:/ / eeas.europa.eu/na/index_es.htm

\section{Entrevistas}

Entrevista con Teresa Barba, Coordinadora de Cooperación para el Desarrollo para Brasil y América Central, Comisión Europea, DG DEvco, Bruselas, 27 de mayo de 2014.

Entrevista con Adrianus Koetsenruijter, Encargado de la División de países del Mercosur, seae, Bruselas, 27 de mayo de 2014.

Entrevista con Neal Mac Call, Desk Officer para Argentina, SEAE, Bruselas, $18 / 06 / 2014$.

Entrevista con Ignacio Sobrino-Castello, Desk Officer para Brasil, SEAE, Bruselas, 18 de junio de 2014.

Entrevista con Philipp Oliver Gross, Responsable de Relaciones Internacionales para México, SEAE, Bruselas, 18 de junio de 2014.

Entrevista con Branko Andjic, Asesor de Prensa, SEAE, Buenos Aires, 22 de juliom de 2014.

Entrevista con Humberto Netto, Asesor de Prensa, seAe, Brasilia, 8 de agosto de 2014.

Entrevista con Rita Junqueira, Sección Política, Económica y de Comunicación, SEAE, Brasilia, 8 de agosto de 2014. 
Entrevista con Erik Von Pistohlkors, Sección Política, Económica y de Comunicación, SEAE, Brasilia, 08/08/2014.

Entrevista con Ekaterini Makri, Asesora de Prensa, SEAE, ciudad de México, 15 de agosto de 2014. 